ORIGINAL ARTICLE

\title{
The binding of proteinase 3 antineutrophil cytoplasmic antibodies (PR3-ANCA) varies in different ELISAs
}

\author{
M Trevisin, P Neeson, J Savige
}

J Clin Pathol 2004;57:303-308. doi: 10.1136/¡cp.2003.009332

See end of article for authors' affiliations

Correspondence to: Professor J Savige,

University of Melbourne, Department of Medicine, Austin Health/Northern Health, The Northern Hospital, Epping VIC 3076, Australia; jasavige@unimelb.edu.au

Accepted for publication 28 July 2003
Background: The demonstration of proteinase 3 specific antineutrophil cytoplasmic antibodies (PR3ANCA), and the estimation of antibody values are useful in the diagnosis and management of patients with Wegener's granulomatosis (WG). However, external quality assessment programmes suggest that PR3-ANCA binding varies in different assays.

Aim: To demonstrate variations in PR3-ANCA binding in different commercial and in house enzyme linked immunosorbent assays (ELISAs).

Method: Binding of a PR3-ANCA standard and 19 sera from patients with WG was compared in eight commercial and in house assays. Binding was expressed in different units depending on the kit.

Results: One commercial assay performed unsatisfactorily. Three commercial kits produced PR3-ANCA binding $(70,102$, and $84 \mathrm{U} / \mathrm{ml})$ close to the expected value for the standard (100 U/ml). Serial dilutions of this standard were linear in only one commercial assay and the in house assay. Sera from patients with WG with borderline binding in the in house assay bound in the eight commercial kits at 0-148 kit units; low binding sera ranged from 0 to 273 units; moderately strong sera bound at 7-260 units; and strongly binding sera bound at 13-336 units. In four assays, at least one strongly positive serum bound at levels greater than the provided range.

Conclusions: Levels of antibody binding and units of binding have not been standardised in commercially available PR3-ANCA ELISAs. This may affect the diagnosis and management of patients with WG, in addition to the implementation of international guidelines for treatment.
A ntineutrophil cytoplasmic antibodies (ANCA) are autoantibodies directed against antigens in the cytoplasmic granules of neutrophils and monocytes. ${ }^{12}$ The demonstration of these antibodies is useful in the diagnosis and management of patients with Wegener's granulomatosis and microscopic polyangiitis, ${ }^{23}$ who may develop kidney failure if undiagnosed or inadequately treated.

The "International Consensus Statement on Testing and Reporting $\mathrm{ANCA}^{\prime 4}$ requires all laboratories to screen for ANCA by indirect immunofluorescence (IIF) and to confirm cytoplasmic (C-ANCA) or perinuclear (P-ANCA) staining in enzyme linked immunosorbent assays (ELISAs) for antibodies against proteinase 3 (PR3) and myeloperoxidase (MPO). ${ }^{56}$ Typically C-ANCA with PR3 specificity occurs in Wegener's granulomatosis, and P-ANCA directed against MPO are found in microscopic polyangiitis. In addition to the demonstration of the antibody, estimations of antibody values are helpful in the diagnosis and management of these conditions. Antibody values are usually high at presentation, fall with treatment, and increase before relapse. ${ }^{7}$ Determining the antibody value by IIF titration is laborious and expensive, but ELISAs automatically measure the amount of binding, in addition to confirming the IIF positivity.

"The demonstration of antineutrophil cytoplasmic antibodies is useful in the diagnosis and management of patients with Wegener's granulomatosis and microscopic polyangiitis"

We have shown previously that IIF patterns vary in different laboratories, usually as a result of the use of different substrates, rather than errors in interpretation. ${ }^{8}$ The external quality assurance programme of the Royal College of Pathologists of Australasia suggests that binding in PR3ANCA assays also varies in different laboratories. This may interfere with the diagnosis of Wegener's granulomatosis, which depends on the demonstration of high antibody values, and may also hinder the ability to monitor disease activity accurately in patients tested consecutively at different sites. Such variation will thus interfere with the implementation of guidelines for the diagnosis, treatment, and prediction of relapse, which incorporate ANCA values.

The aims of our study were to determine the amount of variation in antibody binding of an internationally available PR3-ANCA standard and of sera from patients with Wegener's granulomatosis in different PR3-ANCA assays.

\section{METHODS \\ IIF for ANCA}

Sera were tested at a 1/20 dilution on commercial neutrophil slides (INOVA Diagnostics, San Diego, California, USA), followed by washing and incubation with 1/100 fluorescein isothiocyanate labelled anti-IgG (Silenus; Chemicon Australia, Victoria, Australia). Slides were then examined using a Leitz LaborLux 12 microscope at $\times 400$ magnification. Each batch of sera was tested with negative and positive controls, and the presence of neutrophil immunofluorescence and the specific patterns were recorded.

In house and commercial ELISAs for PR3-ANCA

The in house ELISA has been modified from the assay

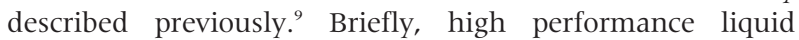
chromatography purified PR3 (Wieslab, Lund, Sweden) was

Abbreviations: ANCA, antineutrophil cytoplasmic antibodies; ANA, antinuclear antibodies; C, cyłoplasmic; ELISA, enzyme linked immunosorbent assay; IIF, indirect immunofluorescence; MPO, myeloperoxidase; P, perinuclear; PBS, phosphate buffered saline; PR3, proteinase 3; SSI, Statens Seruminstitut; T20, Tween 20 
Table 1 Characteristics of the commercial $(\mathrm{A}-\mathrm{H})$ and in house assays for PR3-ANCA

\begin{tabular}{|c|c|c|c|c|c|c|c|c|}
\hline \multirow[b]{2}{*}{ Kit } & \multirow[b]{2}{*}{ Units } & \multirow{2}{*}{$\begin{array}{l}\text { Number of } \\
\text { standards }\end{array}$} & \multirow[b]{2}{*}{ Dynamic range } & \multirow{2}{*}{$\begin{array}{l}\text { Sample } \\
\text { dilution }\end{array}$} & \multirow[b]{2}{*}{ Data reduction } & \multicolumn{3}{|c|}{ Assay performance } \\
\hline & & & & & & Sample & Intra-assay CV\% & Interassay CV\% \\
\hline \multirow[t]{3}{*}{ A } & Units & Low pos & & $1 / 101$ & $\begin{array}{l}\text { (OD samples/OD low } \\
\text { positive) } \times \text { ELISA low } \\
\text { positive }\end{array}$ & Negative & 3 & 4 \\
\hline & & & & & & Low & 2 & 9 \\
\hline & & & & & & High & 3 & 2 \\
\hline \multirow[t]{3}{*}{ B } & $\mathrm{U} / \mathrm{ml}$ & 6 & $0-100$ & $1 / 100$ & Log/linear 4 parameter & 1 & 4.7 & 6.2 \\
\hline & & & & & & 2 & 2.8 & 8.8 \\
\hline & & & & & & 3 & 2.8 & 3.9 \\
\hline \multirow[t]{2}{*}{ C } & $\mathrm{U} / \mathrm{ml}$ & 7 & $0-100$ & $1 / 51$ & Best fit curve & 1 & 6.3 & 7.3 \\
\hline & & & & & & 2 & 6.8 & 9.6 \\
\hline D & Units & 5 & $24-305$ & $1 / 40$ & Linear best fit curve & - & - & - \\
\hline \multirow[t]{3}{*}{$\mathrm{E}$} & $\mathrm{U} / \mathrm{ml}$ & 6 & $0-100$ & $1 / 101$ & $\begin{array}{l}\text { Linear plot of normalised } \\
\text { OD: OD mean/OD max } \\
\text { standard \% }\end{array}$ & 1 & 13.3 & 7.6 \\
\hline & & & & & & 2 & 12.1 & 5.6 \\
\hline & & & & & & 3 & 13.9 & 5.3 \\
\hline \multirow[t]{2}{*}{$\mathrm{F}$} & $\mathrm{EU} / \mathrm{ml}$ & 4 & $21-170$ & $1 / 101$ & $\begin{array}{l}\text { Linear/linear best fit } \\
\text { curve }\end{array}$ & 1 & 10.6 & 8.4 \\
\hline & & & & & & 2 & 6.5 & 8.7 \\
\hline \multirow{4}{*}{ G } & EU & 4 & $10-300$ & $1 / 50$ & Linear/linear & Low & 10 & 14.7 \\
\hline & & & & & & Medium & 2.1 & 8 \\
\hline & & & & & & High & 12.4 & 11.9 \\
\hline & & & & & & Negative & 0 & 0 \\
\hline \multirow[t]{3}{*}{$\mathrm{H}$} & $\mathrm{AU} / \mathrm{ml}$ & 5 & $5-100$ & $1 / 50$ & Log/linear & Low & 8.1 & 9 \\
\hline & & & & & & Medium & 4.7 & 5.2 \\
\hline & & & & & & High & 2.6 & 5.7 \\
\hline \multirow[t]{2}{*}{$\mathrm{I} / \mathrm{H}$} & $\mathrm{U} / \mathrm{ml}$ & 8 & $0-100$ & $1 / 50$ & Log/linear 4 parameter & Low & 5 & 7 \\
\hline & & & & & & High & 21 & 24 \\
\hline
\end{tabular}

coated on to microtitre plates (Costar, Corning, New York, USA) at $0.1 \mu \mathrm{g} / \mathrm{ml}$ in phosphate buffered saline (PBS) by overnight incubation at $37^{\circ} \mathrm{C}$. The plates were then washed $\times 4$ in PBS/Tween 20 (Sigma-Aldrich, Steinheim, Germany) (PBS/T20), and the wells were blocked with $0.1 \%$ human serum albumin (CSL, Parkville, Victoria, Australia) for one hour at $37^{\circ} \mathrm{C}$. Plates were washed four times in PBS/T20 and used within 48 hours. Sera and controls were tested in duplicate at $1 / 50$ in $\mathrm{PBS} / \mathrm{T} 20$ and each assay contained positive and negative controls. Bound antibody was detected with alkaline phosphatase linked antihuman IgG (Silenus) diluted $1 / 500$ in PBS/T20 containing $0.1 \%$ bovine serum albumin (CSL). All incubations were for one hour at $37^{\circ} \mathrm{C}$, and plates were washed four times with PBS/T20. The substrate $p$-nitrophenyl phosphate (Sigma) was then added,

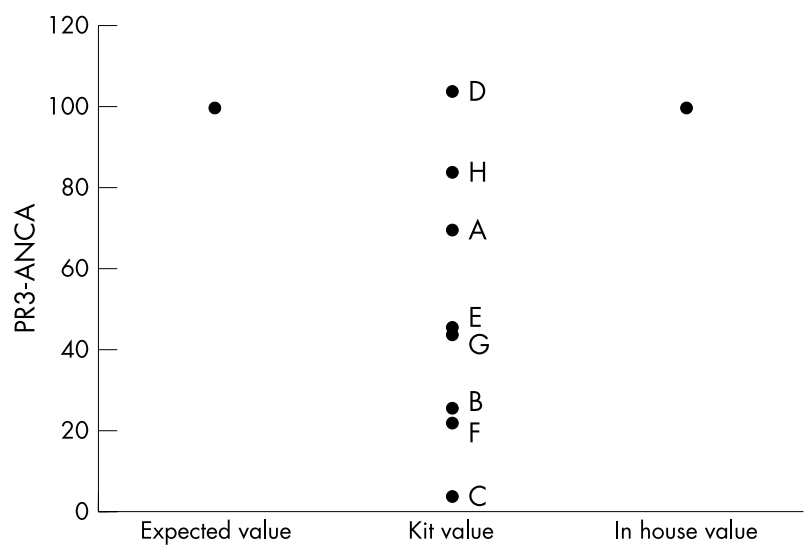

Figure 1 Binding of the SSI standard in the commercial (A-H) and in house assays for proteinase 3 specific antineutrophil cytoplasmic antibodies (PR3-ANCA). Binding of the standard (expected value $100 \mathrm{U} / \mathrm{ml}$ ) varied from $4 \mathrm{U} / \mathrm{ml}$ (kit C) to $102 \mathrm{U} / \mathrm{ml}$ (kit D). The in house assay has been developed so that the standard binds at $100 \mathrm{U} / \mathrm{ml}$. and the colour development stopped after 15 minutes at $37^{\circ} \mathrm{C}$ with $2 \mathrm{M} \mathrm{NaOH}$. The plates were read at $405 \mathrm{~nm}$ on an ELISA plate reader (Molecular Devices, Sunnyvale, California, USA) using SOFTMAX.Pro for Windows software. Positive sera were defined as those that bound at levels greater than mean +3 SD of 40 hospital patients who were ANCA negative by IIF.

Table 1 shows the features of the eight commercial kits (A$\mathrm{H})$ and the in house assay, including the intra-assay and interassay coefficients of variation, supplied by the manufacturers. Kits were provided by the manufacturers without charge and without restriction on their use. Assays were performed as recommended by the manufacturers. The sera and standard were diluted $1 / 100$ in kits $\mathrm{A}, \mathrm{B}, \mathrm{E}$, and $\mathrm{F} ; \mathrm{l} / 50$ in kits $C, G$, and $H$; and $1 / 40$ in kit $D$. The number of standards varied from one to seven in each kit, and their corresponding values varied from 0-100 to 24-305. Results were calculated in various ways. Kit A supplied one standard that was used to produce a ratio using the standard value and the optical

Table 2 Binding of doubling dilutions of the SSI standard in the commercial $(\mathrm{A}-\mathrm{H})$ and in house assays for PR3ANCA

\begin{tabular}{|c|c|c|c|c|c|c|c|c|c|}
\hline \multirow{2}{*}{$\begin{array}{l}\text { Expected } \\
\text { value }\end{array}$} & \multicolumn{9}{|c|}{ Actual value } \\
\hline & $\mathrm{I} / \mathrm{H}$ & A & B & C & D & $\mathbf{E}$ & $\mathbf{F}$ & G & H \\
\hline 2 & 0 & 5 & 0 & 0.0 & 17 & 0 & 6 & 0.0 & 3 \\
\hline 3 & 3 & 11 & 2 & 0.1 & 18 & 0 & 7 & 0.2 & 4 \\
\hline 6 & 7 & 17 & 4 & 0.2 & 19 & 0 & 8 & 0.5 & 5 \\
\hline 13 & 13 & 28 & 7 & 0.4 & 22 & 7 & 10 & 1.9 & 10 \\
\hline 25 & 24 & 45 & 13 & 0.9 & 28 & 24 & 14 & 6.6 & 22 \\
\hline 50 & 52 & 70 & 25 & 1.8 & 41 & 46 & 23 & 21.7 & 44 \\
\hline 100 & 100 & 100 & 55 & 3.8 & 67 & 75 & 38 & 44.0 & 84 \\
\hline
\end{tabular}

Binding was linear only with kit B (although binding was low) and the in house assay. Kit $C$ did not perform satisfactorily. Binding is shown graphically in fig 3 .

$\mathrm{I} / \mathrm{H}$, in house. 

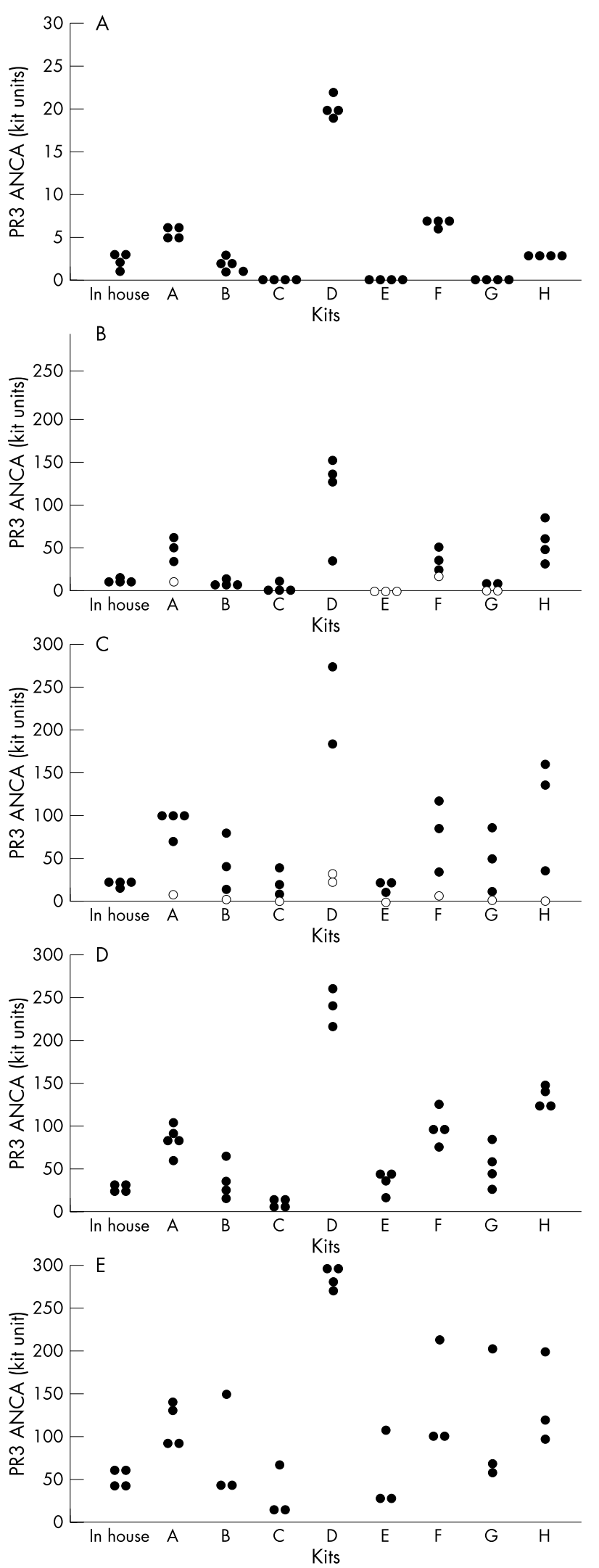

Figure 2 Binding of sera from (A) antineutrophil cytoplasmic antibody (ANCA) negative hospital patients thought to have vasculitis and (B-E) from patients with Wegener's granulomatosis in the commercial $(\mathrm{A}-\mathrm{H})$ and in house assays for proteinase 3 specific ANCA (PR3-ANCA).

Positive test sera from patients with Wegener's granulomatosis bound within ranges considered to be (B) borderline, (C) low, (D) moderate, or (E) strong in the in house assay (closed circles). Sera with negative PR3- density of the patient sample. Kit $\mathrm{C}$ used the line of best fit. Kit $\mathrm{E}$ required a calculation of normalised optical densities for each standard and patient sample to produce a standard curve. Kit $\mathrm{G}$ required the assay to be read at times 0 and 60 minutes, and the difference in optical densities to be calculated and plotted against the concentration using a computer program.

The results were expressed in the units used by the individual kits. All ELISA kits supplied a cut off value for negative sera. Kits A, F, and G supplied ranges for borderline to strong positive results. Kit E supplied a negative cutoff and an equivocal range where repeat testing was recommended. All kits except $C$ and $G$ suggested that laboratories should establish their own cutoff point and pathological ranges for PR3-ANCA.

\section{PR3-ANCA serum standard}

The PR3-ANCA positive serum standard was obtained from the Statens Seruminstitut (SSI), Copenhagen, Denmark. This serum was from a patient with active small vessel vasculitis. It produced a C-ANCA IIF pattern at a 1/320 dilution, and had PR3 specificity in an ELISA using human neutrophil $\alpha$ granule substrate. It was also weakly positive for IgM and IgA rheumatoid factor, in addition to containing anti-striated heart muscle antibodies.

The lyophilised standard was reconstituted with $200 \mu \mathrm{l}$ distilled $\mathrm{H}_{2} \mathrm{O}$ and diluted $1 / 10$ as recommended by the SSI. This was said to be equivalent to a PR3-ANCA value of $100 \mathrm{U} /$ $\mathrm{ml}$ when assayed at a 1/50 dilution in their in house assay. The manufacturer of kit $\mathrm{E}$ indicated in their packet insert that they used this standard in the development of their PR3ANCA ELISA, and it was also used to standardise binding results in the in house assay.

\section{Binding of serial dilutions of the PR3-ANCA standard in different ELISAs}

The SSI PR3-ANCA standard was diluted for testing according to the instructions in individual kits ( $1 / 40$ to $1 /$ 100), and this dilution together with subsequent doubling dilutions were assayed in each of the eight commercial PR3ANCA ELISAs and the results expressed in individual kit units.

\section{Identification of sera with varying PR3-ANCA values in the in house ELISA}

The normal range of the in house PR3-ANCA ELISA was calculated from the mean +3 SD of the serum binding of 40 hospital patients suspected clinically to have vasculitis, but who were negative for ANCA by IIF. These sera were also negative for antinuclear antibodies (ANA) and MPO-ANCA by an in house ELISA. ${ }^{10}$ Binding was calculated in $\mathrm{U} / \mathrm{ml}$, and the mean and SD were $3 \mathrm{U} / \mathrm{ml}$ and $1 \mathrm{U} / \mathrm{ml}$, respectively, resulting in a normal range of $\leqslant 6 \mathrm{U} / \mathrm{ml}($ mean $+3 \mathrm{SD})$. Four of these sera were chosen for testing in the eight commercial ELISAs.

Sera from 18 patients with recent onset Wegener's granulomatosis diagnosed clinically or histologically, and from 34 patients with treated or convalescent disease who were positive for C-ANCA (but not for ANA or P-ANCA), were examined for binding in the in house PR3-ANCA ELISA and the results expressed in $\mathrm{U} / \mathrm{ml}$. Six of these sera were negative, and the others bound at 7-100 U/ml (where $100 \mathrm{U} /$ $\mathrm{ml}$ was the upper limit of binding in the standard curve). Fourteen of the 18 sera from patients with active generalised Wegener's granulomatosis bound at $>34 \mathrm{U} / \mathrm{ml}$, which was

ANCA binding are indicated by open circles. Binding is expressed in individual kit units. The amount of binding for individual sera was very different in the different assays tested. 


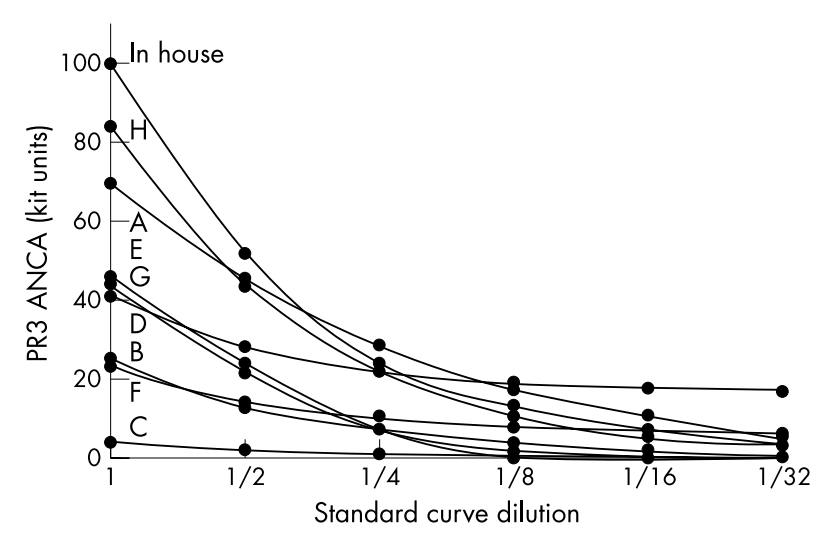

Figure 3 Binding of doubling dilutions of the Statens Seruminstitut standard in the commercial $(A-H)$ and in house assays for proteinase 3 specific antineutrophil cytoplasmic antibodies (PR3-ANCA). Binding was linear only with kit B (but binding levels were low) and the in house assay. Actual binding is shown in individual kit units in table 2 .

considered to be strong binding. Positive binding below this value was divided into three ranges. Binding of $7-14 \mathrm{U} / \mathrm{ml}$ was considered borderline; 15-22 U/ml low; and 23-33 U/ml moderate. Fifteen of these sera were chosen for testing in the eight commercial ELISAs.

\section{Binding of sera with varying PR3-ANCA values in the commercial ELISAs}

The four negative sera and the 15 sera from patients with Wegener's granulomatosis where binding was borderline (BL1-BL4), low (Ll-L4), moderate (Ml-M4), or strong (S1$\mathrm{S} 3$ ) in the in house PR3-ANCA ELISA were then tested in the eight commercial PR3-ANCA ELISAs.

\section{RESULTS}

Performance of commercial PR3-ANCA ELISAs (table 2, figs 2 and 3 )

All kits performed satisfactorily except for kit C. In kit C, binding was very low with doubling dilutions of the SSI standard, and two of the three sera that bound strongly in the in house assay bound at less than $20 \mathrm{U} / \mathrm{ml}$.

\section{Binding of serial dilutions of the PR3-ANCA standard in different ELISAs}

The SSI PR3-ANCA standard bound in the commercial assays at values ranging from 4 to $102 \mathrm{U} / \mathrm{ml}$ (fig 1). Only kits $\mathrm{A}, \mathrm{D}$, and $\mathrm{H}$, in which sera were tested at dilutions of $1 / 100,1 / 40$, and $\mathrm{l} / 50$ and produced results of $70 \mathrm{U}, 102 \mathrm{U}$, and $84 \mathrm{AU} / \mathrm{ml}$, respectively, were close to the expected value of $100 \mathrm{U} / \mathrm{ml}$.

Serial dilutions of the SSI PR3-ANCA standard demonstrated poor linearity for PR3-ANCA binding in all kits except $\mathrm{B}$ and the in house assay (table 2; fig 3). Binding was linear in kit $\mathrm{C}$ but antibody values were very low.

\section{Binding of sera with varying PR3-ANCA values in the commercial ELISAs}

All eight commercial assays showed an overall increase in PR3-ANCA binding of the 15 sera from patients with Wegener's granulomatosis from borderline through to low, moderate, and strong binding (fig 2). However, the in house assay showed relatively little variation between low (15$22 \mathrm{U} / \mathrm{ml}$ ) and strongly (> $34 \mathrm{U} / \mathrm{ml}$ ) binding sera, and in the commercial ELISAs the range of binding was least in kit $\mathrm{C}$ (with a minimum of $4 \mathrm{U} / \mathrm{ml}$ and maximum of $67 \mathrm{U} / \mathrm{ml}$ ) and greatest with kit D (with a minimum of $24 \mathrm{U}$ and maximum of $305 \mathrm{U}$ ). The commercial assays did not provide ranges for low, moderate, or strong binding and it was thus not possible to compare the binding values meaningfully between kits.

All four sera that were negative for PR3-ANCA in the in house ELISA were negative in each of the eight commercial assays. However, four kits (A, E, F, and G) also found BL2 negative, and two kits found two additional BL sera to be negative (BLl and BL3 in kit E, and BL3 and BL4 in kit G). Kit D found L1 negative also, and L3 (expected value of $19 \mathrm{U} / \mathrm{ml}$ ) was negative in all eight kits.

Negative sera bound at less than $7 \mathrm{U} / \mathrm{ml}$ in all kits except $\mathrm{D}$, where binding was $>20 \mathrm{U}$. In the eight commercial kits, sera with borderline binding in the in house assay (BLl-BL4, with binding 9-14 $\mathrm{U} / \mathrm{ml}$ in the in house assay) bound at 0 148 units; low binding sera (L1-L4, with expected values 16$22 \mathrm{U} / \mathrm{ml}$ ) ranged from 0 to 273 units; moderately strong sera (MI-M4, with expected values $25-30 \mathrm{U} / \mathrm{ml}$ ) bound at 7-260 units; and strongly binding sera (S1-S3, with expected values $41-61 \mathrm{U} / \mathrm{ml}$ ) bound from 13 to 336 units.

Kits were also evaluated for the binding of individual sera. Kit A found BL2 to be negative, but showed strong binding for BL1, BL3, Ll, L2, and L4. Kit B bound $\mathrm{S} 1$ at a value higher than the highest standard used to calculate binding. Kit $\mathrm{C}$ bound all sera weakly, with M2, M3, and M4 binding at 6$9 \mathrm{U} / \mathrm{ml}$, and S2 and S3 at 13 and $18 \mathrm{U} / \mathrm{ml}$, respectively. Kit D was the only one to find Ll negative, and this kit also showed strong binding of BL1, BL3, BL4, L2, and L4, but these were still within the range of the standard curve. Kit $\mathrm{E}$ found that BL1, BL2, and BL3 were all negative, and all kits except E found significantly raised PR3-ANCA values for at least two low controls. In addition, in kit E, S1 produced strong binding - greater than the highest standard. Kit F found BL2 to be negative, and Sl also bound more strongly than the highest standard. Kit G found BL2, BL3, and BL4 all negative. With kit $\mathrm{H}, \mathrm{BL2}, \mathrm{BL} 3$, and BL4 bound more strongly than with the other kits, and Ml-M4, and again S1 and S3, bound more strongly than the highest standard.

The in house assay found L3 to be positive, but all eight commercial kits found it negative, and although L1 and L2 bound within the low range in the in house assay, six of the eight commercial kits found stronger binding. The clinical notes of the patient corresponding to L3 were then reviewed and interestingly this patient's diagnosis had been revised from Wegener's granulomatosis.

\section{DISCUSSION}

Many previous studies of ANCA testing have been concerned with the sensitivity and specificity of IIF alone or together with PR3-ANCA ELISA in the diagnosis of Wegener's granulomatosis. ${ }^{11-16}$ Some studies have compared different commercial and in house IIF and ELISA methods to find the most sensitive and specific assays, ${ }^{14-16}$ and others have standardised IIF and ELISA testing in in house assays to improve reproducibility. ${ }^{1-16}$ All studies have focused on diagnostic testing for Wegener's granulomatosis and the ability of assays to distinguish between patients with or without the disease. However ANCA testing is also used to follow the response to treatment and to indicate relapse, and antibody values are important in patient management. None of the previous studies has examined ANCA binding values in different assays. In contrast, in the study described here we compared the amount of PR3-ANCA binding in commercial assays and an in house ELISA of a commonly used serum standard and sera from patients with active and treated Wegener's granulomatosis. It did not matter that one of these patients was subsequently shown to have a disease other than Wegener's granulomatosis because the test sera were chosen to examine the range of binding, rather than sensitivity and specificity in the different ANCA assays. 
In the first part of our study we compared the binding of the PR3-ANCA standard in eight commercially available ELISAs when tested at dilutions recommended by the manufacturers. This standard bound at widely different values. Furthermore, binding of serial dilutions of this standard was linear in only one commercial assay and the in house ELISA.

In the second part of our study, we examined the PR3ANCA binding of sera from patients with active and treated Wegener's granulomatosis in the commercial assays, where their level of binding had already been assessed in the in house assay. We also tested the PR3-ANCA standard from the SSI. We used this even though it is not a true reference standard because it is the best that we have and many testing laboratories are familiar with it. The in house ELISA performed satisfactorily in that all negative sera from patients were also negative in the eight commercial assays, and all positive sera were also positive in at least four and usually all eight commercial assays. In addition, the average binding of sera in each category-negative through to strongly positive-in the in house assay increased in all eight commercial ELISAs (except in kit C). However, one serum that bound weakly in the in house ELISA was negative in all the commercial kits, and two other sera that bound weakly bound more strongly in the commercial assays.

"The amount of binding in kit units of the Statens Seruminstitut proteinase 3 specific antineutrophil cytoplasmic antibodies standard and of the negative and positive patient sera varied in the different commercial assays"

All eight commercial assays identified the sera that were negative for PR3-ANCA, but five found at least one serum that was positive in most of the assays to be negative. Several sera produced binding in the commercial assays that was greater than their highest standard, so that these results could not be assigned exact values. However, the amount of binding in kit units of the SSI PR3-ANCA standard and of the negative and positive patient sera varied in the different commercial assays. Some of this variation will have resulted from the use of the different testing dilutions, methods of PR3 purification, binding properties of the ELISA plates, buffers used for coating and incubation, negative and positive controls, and methods used to construct standard curves and calculate PR3-ANCA binding. None of the commercial assays had attempted to make their assays semiquantitative by indicating that binding was low, moderate, or strong.

\section{Take home messages}

- Commercial assays for assessing proteinase 3 specific antineutrophil cytoplasmic antibodies (PR3-ANCA) have been optimised for distinguishing between sera that are negative and positive for PR3-ANCA, but have not been standardised to allow their results to be compared

- This may affect the diagnosis and management of patients with Wegener's granulomatosis, in addition to the implementation of international guidelines for treatment

- Standardisation of these assays using an internationally available standard would be useful to help remedy this situation
These observations suggest that the commercial assays have been optimised to distinguish between sera that are negative and positive for PR3-ANCA, but have not been standardised to allow their results to be compared. (Only one commercial assay had used the SSI PR3-ANCA standard in its development.)

We have not indicated the kit that performed "best" in our study. This is because we did not perform a kit evaluation but rather compared PR3-ANCA binding in different kits. Furthermore, at least one of the manufacturers has subsequently modified their assay characteristics.

We have shown here how much the binding of a PR3ANCA standard and of PR3-ANCA sera from patients with Wegener's granulomatosis varies between different commercially available assays. It is more convenient to measure antibody values in an ELISA than with IIF, and the international consensus statement suggests that patients previously positive for ANCA by IIF or ELISA can be followed by ELISA antibody values alone. However, standardisation of these assays using an internationally available standard is important if we are to incorporate PR3-ANCA and MPOANCA values into guidelines for the diagnosis, treatment, or prediction of relapse in Wegener's granulomatosis, which will ultimately improve the ability of clinicians to care for their patients with this disease.

The production of an MPO-ANCA standard and standardisation of the MPO-ANCA ELISA is also important because low MPO-ANCA values occur in several non-vasculitic autoimmune diseases, which are often distinguished from active microscopic polyangiitis only by antibody values.

\section{ACKNOWLEDGEMENTS}

We would like to thank the manufacturers who all provided complimentary testing kits with no restrictions on their use.

\section{Authors' affiliations}

M Trevisin, P Neeson, Immunology Unit, Division of Laboratory Medicine, Austin Hospital, Heidelberg, Victoria 3084, Australia J Savige, University of Melbourne, Department of Medicine, Austin Health/Northern Health, The Northern Hospital, Epping VIC 3076, Australia

\section{REFERENCES}

1 Davies DJ, Moran JE, Niall JF, et al. Segmental necrotising glomerulonephritis with antineutrophil antibody: possible arbovirus aetiology? BMJ 1982;285:606

2 van der Woude FJ, Rasmussen N, Lobatto S, et al. Autoantibodies against neutrophils and monocytes: tool for diagnosis and marker of disease activity in Wegener's granulomatosis. Lancet 1985:1:425-9.

3 Savage COS, Winearls CG, Jones S, et al. Prospective study of radioimmunoassay for antibodies against neutrophil cytoplasm in diagnosis of systemic vasculitis. Lancet. 1987;i:1389-93.

4 Savige J, Gillis D, Benson E, et al. International consensus statement on testing and reporting of antineutrophil cytoplasmic antibodies (ANCA). Am J Clin Pathol 1999; 111 :507-13.

5 Goldschmeding $\mathbf{R}$, van der Schoot CE, ten Bokkel Huinink D, et al. Wegener's granulomatosis autoantibodies identify a novel diisopropylfluorophosphatebinding protein in the lysosomes of normal human neutrophils. $J$ Clin Invest 1988;4:1577-87

6 Falk RJ, Jennette JC. Antineutrophil cytoplasmic autoantibodies with specificity for myeloperoxidase in patients with systemic vasculitis and idiopathic necrotising and crescentic glomerulonephritis. N Engl J Med 1988;318:1651-7.

7 Cohen Tervaert JW, van der Woude FJ, Fauci AS, et al. Association between active Wegener's granulomatosis and anticytoplasmic antibodies. Arch Intern Med 1989;149:2461-5

8 Pollock W, Clarke K, Gallagher K, et al. Immunofluorescent patterns produced by antineutrophil cytoplasmic antibodies (ANCA) vary depending on neutrophil substrate and conjugate. J Clin Pathol 2002;55:680-3.

9 Jennings JG, Chang L, Savige JA. Antiproteinase 3 antibodies, their characterisation and disease associations. Clin Exp Immunol 1994:95:251-6.

10 Chang L, Binos S, Savige J. Epitope mapping of antiproteinase 3 and antimyeloperoxidase antibodies. Clin Exp Immunol 1995;102:112-19.

11 Hagen EC, Andrassy K, Csernok E, et al. The value of indirect immunofluorescence and solid phase techniques for ANCA detection. A report on the first phase of an international cooperative study on the standardisation of ANCA assays. J Immunol Methods 1993;159:1-16. 
12 Hagen EC Andrassy K, Csernok E, et al. Development and standardisation of solid phase assays for the detection of anti-neutrophil cytoplasmic antibodies (ANCA). A report on the second phase of an international cooperative study on the standardisation of ANCA assays. J Immunol Methods 1996;196:1-15.

13 Hagen EC, Daha MR, Hermans J, et al. Diagnostic value of standardised assays for antineutrophil cyłoplasmic antibodies in idiopathic systemic vasculitis. Kidney Int 1998;53:743-53.

14 Lim LCL, Taylor JG, Schmitz JL, et al. Diagnostic usefulness of antineutrophil cyłoplasmic autoantibody serology. Comparative evaluation of commercial indirect immunofluorescent antibody kits and enzyme immunoassay kits. Am J Clin Pathol 1999;1 11:363-9.

15 Pollock W, Dunster K, Rolland JM, et al. A comparison of commercial and inhouse ELISAs for antineutrophil cytoplasmic antibodies directed against proteinase 3 and myeloperoxidase. Pathology 1999:31:38-43.

16 Csernok E, Ahlquist D, Ullrich S, et al. A critical evaluation of commercial immunoassays for antineutrophil cytoplasmic antibodies directed against proteinase 3 and myeloperoxidase in Wegeners' granulomatosis and microscopic polyangiitis. Rheumatology 2002;41:1313-17. 